\title{
TWO ELEMENTARY COMMUTATIVITY THEOREMS FOR GENERALIZED BOOLEAN RINGS
}

\author{
VISHNU GUPTA \\ Department of Mathematics \\ M.D. University, P.G. Regional Centre \\ Rewari (Haryana), India
}

(Received September 9, 1991 and in revised form April 17, 1992)

\begin{abstract}
In this paper we prove that if $R$ is a ring with 1 as an identity element in which $x^{m}-x^{n} \in Z(R)$ for all $x \in R$ and fixed relatively prime positive integers $m$ and $n$, one of which is even, then $R$ is commutative. Also we prove that if $R$ is a 2-torsion free ring with 1 in which $\left(x^{2^{k}}\right)^{n+1}-\left(x^{2^{k}}\right)^{n} \in Z(R)$ for all $x \in R$ and fixed positive integer $n$ and non-negative integer $k$, then $R$ is commutative.
\end{abstract}

KEY WORDS AND PHRASES. Commutator, 2-torsion free ring. 1991 AMS SUBJECT CLASSIFICATION CODE. 16A70.

\section{INTRODUCTION.}

Throughout this paper, $R$ is an associative ring with 1 as an identity element. We denote the centre of $R$ by $Z(R)$ and the commutator $x y-y x$ by $[x, y]$. Recently, Quadri and Ashraf [1] proved that if $R$ is a ring in which $x^{n+1}-x^{n} \in Z(R)$ for all $x \in R$ and fixed positive integer $n$, then $R$ is commutative. In this paper, we generalize this result.

2. MAIN RESULTS.

We start with the following lemma of Bell [2].

LEMMA 2.1. Let $w \in R$. If for each $x \in R$ there exist relatively prime positive integers $n=n(x)$ and $m=m(x)$ such that

$$
\left[w, x^{n}\right]=\left[w, x^{m}\right]=0, \text { then } w \in Z(R) .
$$

THEOREM 2.1. If $R$ is a ring with $x^{m}-x^{n} \in Z(R)$ for all $x \in R$ and fixed relatively prime positive integers $m$ and $n$, one of which is even, then $R$ is commutative.

PROOF. Let

$$
x^{m}-x^{n} \in Z(R) \quad \text { for all } x \in R \text {. }
$$

Assume $m$ is even and $n$ is odd. Using both $x$ and $-x$ in (2.1) and then adding and subtracting, we get $2 x^{m} \in Z(R)$ and $2 x^{n} \in Z(R)$. Thus $\left[x^{m}, 2 y\right]=\left[x^{n}, 2 y\right]=0$ for all $x, y \in R$; and by Lemma 2.1 $2 y \in Z(R)$ for all $y \in R$. Now we replace $x$ by $x+1$ to obtain

$$
\left[\sum_{r=1}^{m-1}\left({ }_{r}^{m}\right) x^{m-r}, y\right]=\left[\sum_{r=1}^{n-1}\left({ }_{r}^{n}\right) x^{n-r}, y\right]
$$

and since $m$ is even and $n$ is odd and $[2 x, y]=0$, we get $\left[x^{2} p(x)-x, y\right]=0$ for some $p(x) \in Z[x]$. Now 
the theorem follows from Herstein's result [3].

In Theorem 2.1, all the hypotheses are essential. If both $m$ and $n$ are odd or if one of $m$ and $n$ is even and the other odd, but they are not relatively prime; or if both $m$ and $n$ are even; or if $R$ is a ring without the identity element 1 in the hypotheses of the theorem, then $R$ need not be commutative.

EXAMPLE 2.1. It can be shown easily that

$$
R=\left\{\left(\begin{array}{ccc}
a & b & c \\
0 & a & d \\
0 & 0 & a
\end{array}\right): a, b, c, d \in G F(3)\right\}
$$

is a ring with identity element, in which

(i) $x^{3}-x^{9} \in Z(R)$

(ii) $x^{3}-x^{6} \in Z(R)$

(iii) $x^{4}-x^{10} \in Z(R)$

for all $x \in R$, but $R$ is not commutative.

EXAMPLE 2.2.

$$
R=\left\{\left(\begin{array}{ccc}
a & b & c \\
0 & a & d \\
0 & 0 & a
\end{array}\right): a, b, c, d \in G F(2)\right\}
$$

is a ring with identity element in which

(i) $x^{5}-x^{9} \in Z(R)$

(ii) $x^{4}-x^{8} \in Z(R)$

for all $x \in R$, but $R$ is not commutative.

EXAMPLE 2.3.

$$
R=\left\{\left(\begin{array}{ccc}
0 & a & b \\
0 & 0 & c \\
0 & 0 & 0
\end{array}\right): a, b, c, \in G F(3)\right\}
$$

is a ring without identity element with $x^{3}-x^{4} \in Z(R)$ for all $x \in R$, but $R$ is not commutative.

We state the following lemma which can be proved easily.

LEMMA 2.2. If $t=2^{k} n$ where $k$ and $n$ are positive integers, then

$$
\left\{\left(\begin{array}{l}
t+2^{k} \\
(2 r-1)+2^{k}
\end{array}\right)-\left(\begin{array}{l}
t \\
2 r-1
\end{array}\right)\right\} \text { and }\left(\begin{array}{l}
t+2^{k} \\
2 r-1
\end{array}\right) \text { are multiples of } 2^{k} \quad \text { for } r=1,2,3, \cdots \frac{t}{2} .
$$

Now we give the following theorem which generalizes the theorem of Quadri and Ashraf [1] for 2-torsion free rings.

THEOREM 2.2. If $R$ is a 2-torsion free ring with $\left(x^{2^{k}}\right)^{n+1}-\left(x^{2^{k}}\right)^{n} \in Z(R)$ for all $x \in R$ and fixed non-negative integer $k$ and positive integer $n$, then $R$ is commutative.

PROOF. If $k=0$ then result follows from Theorem 2.1. Let $k>0$ and

$$
\left[x^{t+2^{k}}, y\right]=\left[x^{t}, y\right] \text { for all } x, y \in R \text { where } t=2^{k} n
$$

Now we replace $x$ by $x+1$ to obtain 


$$
\left[\sum_{r=1}^{t+2^{k}-1}\left(\begin{array}{c}
t+2^{k} \\
r
\end{array}\right) x^{t+2^{k}-r}, y\right]=\left[\sum_{r=1}^{t-1}\left({ }_{r}^{t}\right) x^{t-r}, y\right]
$$

Next we replace $x$ by $-x$ in (2.2) and subtract the result from (2.2) and use the fact that $R$ is 2 -torsion free to get

$$
\left[\sum_{r=1}^{2^{k-1}}\left(\begin{array}{c}
t+2^{k} \\
2 r-1
\end{array}\right) x^{t+2^{k}-(2 r-1)}+\sum_{r=1}^{t / 2}\left\{\left(\begin{array}{c}
t+2^{k} \\
(2 r-1)+2^{k}
\end{array}\right)-\left(\begin{array}{c}
t \\
2 r-1
\end{array}\right)\right\} x^{t-(2 r-1), y}\right]=0
$$

By Lemma 2.2 and the fact that $R$ is 2 -torsion free, we get $\left[x^{2} p(x)-x, y\right]=0$ for all $x, y \in R$ and some $p(x) \in Z[x]$. Now $R$ is commutative.

All the hypotheses of Theorem 2.2 are essential. In Example 2.1, $R$ is a 2-torsion free ring with identity element in which $\left(x^{2^{k}}\right)^{m}-\left(x^{2^{k}}\right)^{n} \in Z(R)(k=2, m=4, n=7)$ for all $x, y \in R$ and $m$ and $n$ are relatively prime positive integers and one of them is even, but $R$ is not commutative. In Example 2.2, $R$ is a 2-torsion ring with identity element in which $\left(x^{2^{k}}\right)^{n+1}-\left(x^{2^{k}}\right)^{n} \in Z(R)(k=2$, $n=1$ ) for all $x \in R$, but $R$ is not commutative. In Example 2.3, $R$ is a 2-torsion free ring without identity element in which $\left(x^{2^{k}}\right)^{n+1}-\left(x^{2^{k}}\right)^{n} \in Z(R)(k=2$ and $n=1)$ for all $x \in R$, but $R$ is not commutative.

ACKNOWLEDGEMENT. The author expresses his sincere thanks to the referee for many helpful suggestions to modify the proof of Theorem 2.1 .

\section{REFERENCES}

1. QUADRI, M.A. \& ASHRAF, MOHD., Commutativity of generalized Boolean rings, Publ. Math. (Debrecen) 35 (1988), 73-75.

2. BELL, H.E., On rings with commuting powers, Math. Japonica 24 (1979), 473-478.

3. HERSTEIN, I.N., Two remarks on the commutativity of rings, Cand. J. Math. 7 (1955), 411-412.

4. HERSTEIN, I.N., A generalization of theorem of Jacobson, Amer. J. Math. 73 (1951), 755762. 


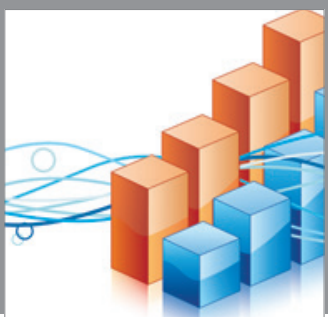

Advances in

Operations Research

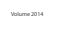

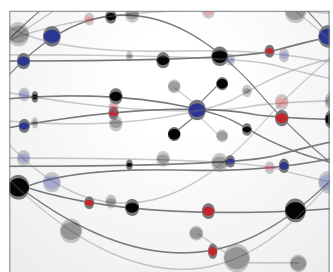

\section{The Scientific} World Journal
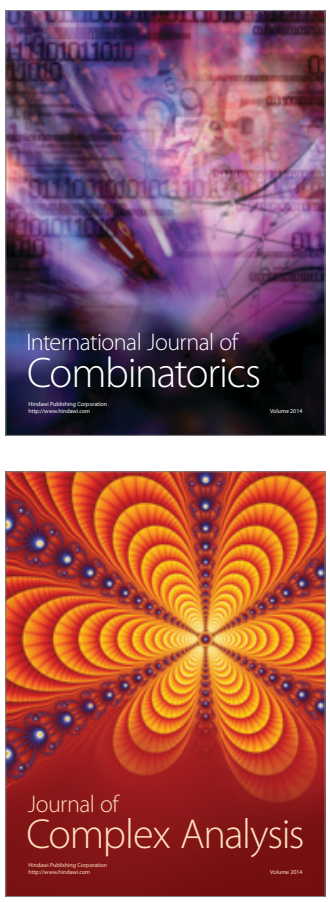

International Journal of

Mathematics and

Mathematical

Sciences
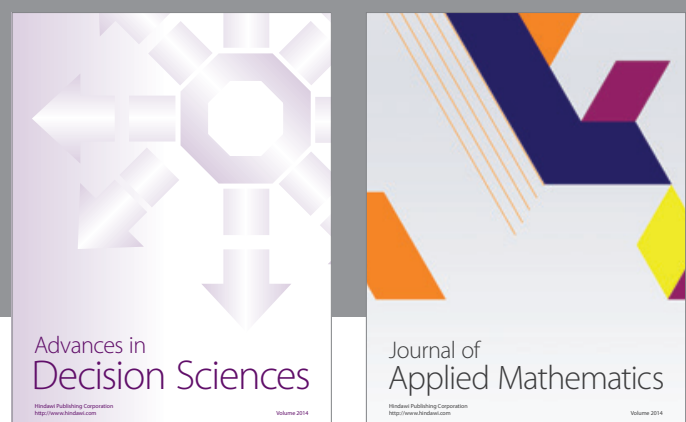

Journal of

Applied Mathematics
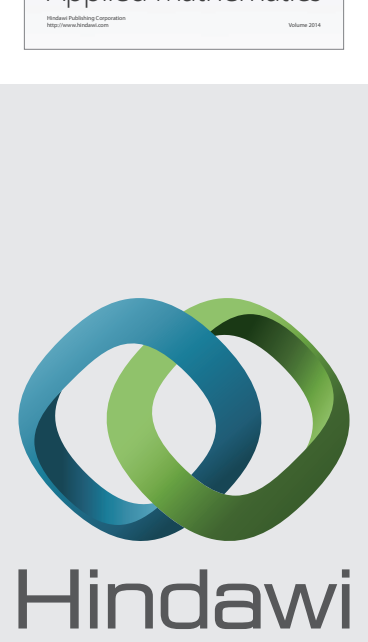

Submit your manuscripts at http://www.hindawi.com
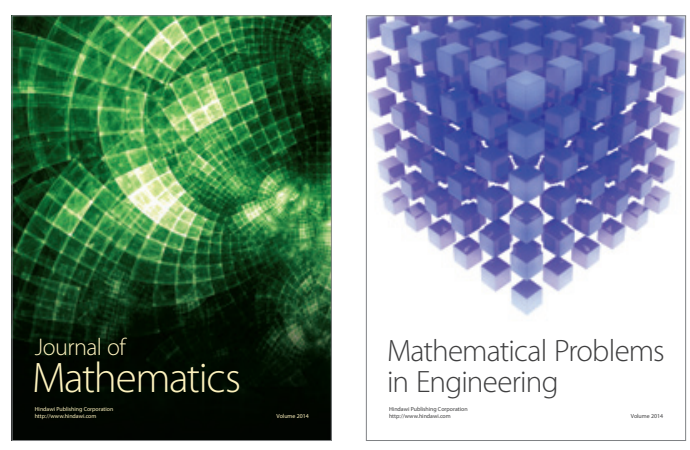

Mathematical Problems in Engineering
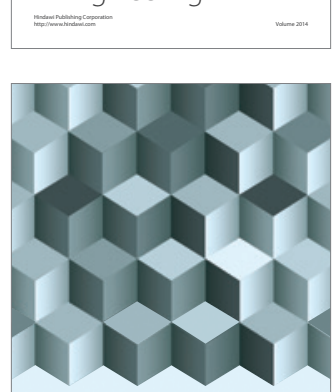

Journal of

Function Spaces
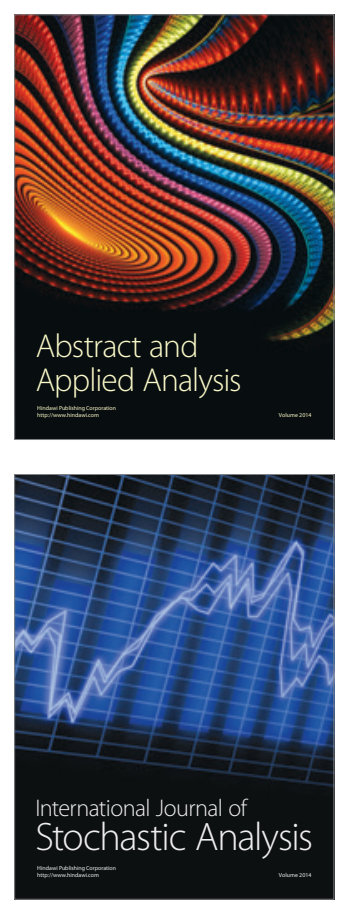

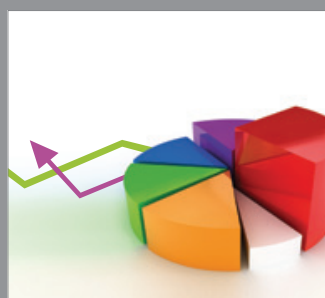

ournal of

Probability and Statistics

Promensencen
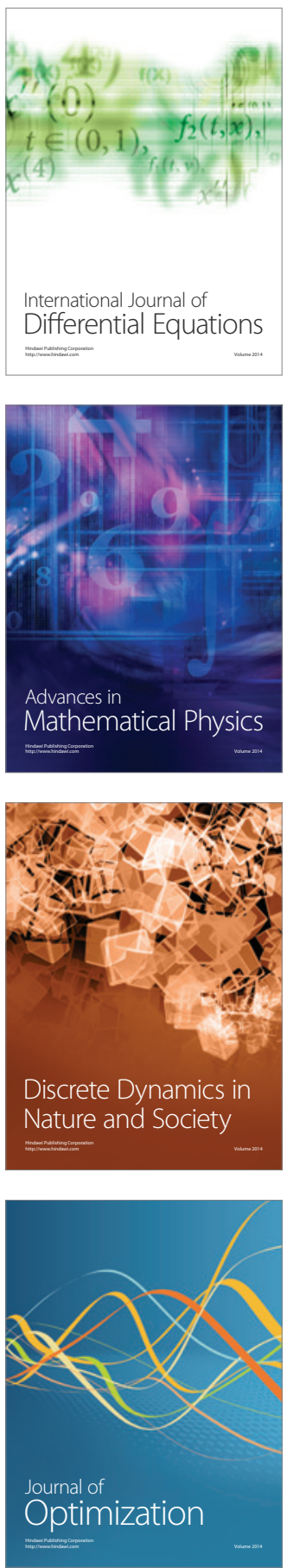